\title{
THE FIBONACCI AUTOMORPHISM OF FREE BURNSIDE GROUPS
}

\author{
Ashot S. PAhlEVANYAN ${ }^{1}$
}

\begin{abstract}
We prove that the Fibonacci morphism is an automorphism of infinite order of free Burnside groups for all odd $n \geq 665$ and even $n=16 k \geq 8000$.
\end{abstract}

Mathematics Subject Classification. 20F28, 20E36, 20F50, $20 \mathrm{M} 05$.

\section{INTRODUCTION}

The question of study of automorphisms of free Burnside groups was stated by Ol'shanskii in the Kourovka Notebook [7]. The first results were obtained by Cherepanov in $[4,5]$ and by Atabekyan in $[2,3]$. In paper [4] it was proved that the Fibonacci morphism is an automorphism of infinite order of free Burnside groups for all odd $n>10^{10}$ and even $n=16 k \geq 8000$.

This paper shows that the bound of odd $n$ can be decreased from $n>10^{10}$ to $n \geq 665$.

Consider an automorphism $\varphi: F_{2} \rightarrow F_{2}$ of the absolutely free group $F_{2}$ of rank two with free generators $\{a, b\}$, given on generators by formulae

$$
\varphi: a \mapsto b, \varphi: b \mapsto a b .
$$

This automorphism is called after Fibonacci since the lengths of words $\varphi^{k}(a)$ are equal to corresponding members of the numerical Fibonacci sequence. If we consider the sequence of mirror copies of words $\varphi^{k}(a)$, we obtain the iterations of the following morphism

$$
h: a \mapsto b, \varphi: b \mapsto b a .
$$

\footnotetext{
Keywords and phrases. Free periodic groups, Burnside groups, group automorphisms, Fibonacci morphism, Fibonacci sequence, Fibonacci word, golden ratio.

${ }^{1}$ Faculty of Mathematics and Mechanics, Yerevan State University, A. Manoogian st. 1, Yerevan-0025, Armenia. apahlevanyan@ysu.am 
This morphism is also called after Fibonacci. All the statements of this paper that we prove for the first morphism hold for the second morphism either.

Automorphism $\varphi$ naturally induces an automorphism of free the Burnside group $B(2, n)$, which we denote by the same letter $\varphi$. Let us remember that a free Burnside group $B(2, n)$ is the quotient $F_{2} / F_{2}{ }^{n}$, where $F_{2}{ }^{n}$ is the subgroup generated by all possible $n$-powers of elements of $F_{2}$. Obviously the group $B(2, n)$ has a presentation $B(2, n)=\left\langle a_{1}, a_{2}\right| A^{n}=1$, for all words $\left.A=A\left(a_{1}, a_{2}\right)\right\rangle$.

Theorem 1.1. For arbitrary odd $n \geq 665$ and arbitrary even $n=16 k \geq 8000$ the Fibonacci automorphism $\varphi$ has infinite order in the group $\operatorname{Aut}(B(2, n))$.

Theorem 1.1 strengthens the similar result of paper [4], decreasing the bound of odd $n$ to $n \geq 665$.

To prove Theorem 1.1 we prove the following result that is individually interesting.

Proposition 1.2. For any natural $k$ no forth power of a non-empty word occurs in a cyclic word $\varphi^{k}(a)$.

As usual, by a cyclic word we mean a word written on a circle without fixing its start. Proposition 1.2 strengthens one of the results of paper [6] by Karhumäki, where a similar statement is proved without the assumption that the word $\varphi^{k}(a)$ is cyclic. Our proof of Proposition 1.2 does not depend on paper [6] by Karhumäki.

Proposition 1.2 also strengthens the Lemma 1.3 of paper [4] by Cherepanov, according to which no 24th power of a non-empty word occurs in a cyclic Fibonacci sequence. Bearing on paper [6] by Karhumäki in [9] Mignosi and Pirillo proved the following interesting result:

Proposition 1.3. The Fibonacci infinite word contains no fractional power with an exponent grater than $2+((\sqrt{5}+1) / 2)$ and, for any real number $\varepsilon>0$, it contains a fractional power with an exponent grater than $2+((\sqrt{5}+1) / 2)-\varepsilon$.

Theorem 1.1 implies

Corollary 1.4. For arbitrary odd $n \geq 665$ the quotient group

$$
\operatorname{Aut}(B(2, n)) / \operatorname{Inn}(B(2, n))
$$

is infinite.

Proof. According to the famous theorem of S. I. Adian (see Thm. VI.3.4 of [1]) the center of $B(m, n)$ is trivial for $n \geq 665$ and $m>1$. Therefore $\operatorname{Inn}(B(2, n))$ is isomorphic to $B(2, n)$. Since any inner automorphism of the group $B(2, n)$ has a finite order, from Theorem 1.1 it follows that for any natural number $l$ each automorphism $\varphi^{l}$ is not inner. Hence the quotient $\operatorname{Aut}(B(2, n)) / \operatorname{Inn}(B(2, n))$ is infinite. 


\section{The Proof of Proposition 1.2}

We adhere to the following notions and notations of monograph [1].

Definition 2.1. The word $A$ is called primitive if it cannot be presented in a form $D^{r}$ for $r>1$.

We say that the word $E$ occurs in a word $X$, if there exist words $R$ and $Q$ such that $X \pm R E Q$ holds. If the word $R$ (word $Q$ ) is empty, then $E$ is a prefix (suffix) of $X$. If $X$ is a word over the alphabet that does not contain the letter $*$ and $X=R E Q$, the word $R * E * Q$ is called an occurrence of word $E$ in a word $X$. $E$ is called a base of the occurrence $R * E * Q$. For a given word $X$ we denote by $\bar{X}$ the cyclic word generated by $X$, that is the word $X$ written on a circle without fixing its start. For a given word $X$ by $\partial(X)$ we denote the length of $X$, that is the number of its letters over the alphabet $\{a, b\}$. For the equality by definition of two words or two occurrences in a same word we use the symbol $\rightleftharpoons$.

Consider an automorphism $\varphi: a \mapsto b, \varphi: b \mapsto a b$ of the group $B(2, n)$. Let us first write out a few images

$$
\varphi^{k}(a): a \mapsto b \mapsto a b \mapsto b a b \mapsto \underbrace{a b} \underbrace{b a b} \mapsto \underbrace{b a b} \underbrace{a b b a b} \mapsto \underbrace{a b b a b} \underbrace{b a b a b b a b} .
$$

Denote

$$
X_{0} \rightleftharpoons \varphi^{0}(a)=a, X_{k} \rightleftharpoons \varphi^{k}(a), k=1,2, \ldots
$$

Since $X_{k+1}=X_{k-1} \cdot X_{k}$, the lengths of words of the sequence $X_{k}, k=1,2, \ldots$ form a Fibonacci sequence. Let us denote

$$
\begin{gathered}
A \rightleftharpoons X_{k}, B \rightleftharpoons X_{k-1}, C \rightleftharpoons X_{k-2}, D \rightleftharpoons X_{k-3}, \\
E \rightleftharpoons X_{k-4}, F \rightleftharpoons X_{k-5}, G \rightleftharpoons X_{k-6}, H \rightleftharpoons X_{k-7} .
\end{gathered}
$$

Then $A=C D C, B=D C, C=E D$ and $X_{k+1}=B A=D C C D C$.

Let us recall the following statements from [1], that we often refer to.

Lemma 2.2 (see Lem. I.2.2 in [1]). If $A B=B A$, then there exists a word $D$, such that $A=D^{t}, B=D^{r}$, for some $t, r \geq 0$.

Lemma 2.3 (see Lem. I.2.9 in [1]). Suppose $A^{t} A_{1}=B^{r} B_{1}$, where $\partial\left(A^{t} A_{1}\right) \geq$ $\partial(A B), A_{1}$ is a suffix of $A, B_{1}$ - a prefix of $B$. If $A$ is a primitive word, then for some $k, B=A^{k}$ holds.

Lemma 2.4 (see Lem. IV.2.16 in [1]). If no elementary $\alpha$-power of rank 1 occurs in a word $X$, then $X \stackrel{\alpha}{\sim} Y \Rightarrow X=Y$.

Definition 2.5 (see Def. I.4.34 in [1]). Suppose $\mathcal{A}=\bigcup_{i=1}^{\infty} \mathcal{A}_{i}$, where

$$
X \in \mathcal{A} \Leftrightarrow X \in \mathcal{R}_{\alpha-1} \& N \operatorname{Norm}(\alpha, X, 9)=\varnothing .
$$

Elements of the set $\mathcal{A}$ are called absolutely reduced. 
From Propositions 1.2 and 1.3 follows

Corollary 2.6. For any natural $k$ the cyclic word $\varphi^{k}(a)$ contains no fractional power $2+((\sqrt{5}+1) / 2)$ of a non-empty word.

Proof. Let us prove it by induction on $k$. The base of induction is obvious. Suppose the statement is true for all natural $l \leq k$ and prove it for $k+1$. Since all cyclic shifts of the word $X_{k+1}=D C C D C$ occur in a Fibonacci word $X_{k+3}=D C C D C C D C D C C D C$ that contains no $2+((\sqrt{5}+1) / 2)$ fractional power according to proposition 1.3 , the word $\overline{X_{k+1}}$ contains no fractional $2+((\sqrt{5}+1) / 2)$ power of a non-empty word either.

Lemma 2.7. None of the words $\overline{X_{k}}$ is a proper power, that is $\overline{X_{k}} \neq Z^{t}, t \geq 2$, for $k=1,2, \ldots$

Proof. Since the cyclic shift of a proper power is itself a proper power, it is enough to prove that $X_{k}$ is not a proper power. We prove this by induction on $k$. For $k \leq 5$ the proof is obvious. Suppose that the lemma is proved for all numbers $l \leq k$, and prove it for $k+1$. We can assume that the word $Z$ is primitive. Let $X_{k+1}=D C C D C=Z^{t}, t \geq 2$. Then $C D C D C=(C D)^{2} C=Z_{1}{ }^{t}$ for some cyclic shift $Z_{1}$ of $Z$. Since $t \geq 2, \partial\left(Z_{1}\right)+\partial(C D)<\partial(C D C D C)$ and by Lemma 2.3 we obtain $\bar{B}=C D=Z_{1}{ }^{p}$. Therefore $B=Z^{p}$ and $A=Z^{q}, p, q \geq 1, p \neq q$. This contradicts the inductive assumption.

Lemma 2.8. If $\overline{X_{k}}$ contains a power $Z^{t}$ then $t<4$.

Proof. The proof is by induction on $k$. For $k \leq 5$ the proof is obvious since the word $\overline{X_{5}}=\overline{b a b a b b a b}$ does not contain $Z^{4}$. For $k=6$ we have the word $\overline{X_{6}}=\overline{a b b a b b a b a b b a b}$ that does not contain a subword $Z^{4}$ with $\partial(Z) \leq 3$. For $k=7$

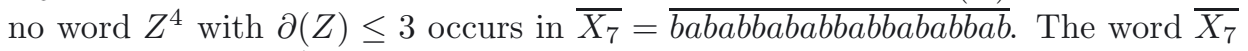
does not contain $Z^{4}$ with $\partial(Z)=4,5$ either. Let $k \geq 7$. Suppose the statement is proved for all $l \leq k$ and prove it for $k+1$. Let $Z^{4}$ occur in $\overline{X_{k+1}}=\overline{D C C D C}$. $Z^{4}$ does not occur in a word $\bar{A}=\overline{C D C}$ by inductive assumption. Since $D$ is a suffix of $C$, any subword of word $\overline{D C C D C}$ of length three over the alphabet $\{C, D\}$ occurs in $\overline{C D C}$. Therefore $Z^{4}$ does not occur in a subword of word $\overline{D C C D C}$ of length three over the alphabet $\{C, D\}$.

Let us prove that $Z^{4}$ does not occur in a subword of $\overline{D C C D C}$ of length four over the alphabet $\{C, D\}$ either. Let us write out subwords of length four of word $\overline{D C C D C}$ over the alphabet $\{C, D\}$ :

$$
D C C D, C C D C, C D C D, D C D C, C D C C \text {. }
$$

Since the word $D C D C$ is a suffix of $C C D C$, it is enough to consider the case of the occurrence of $Z^{4}$ in words $D C C D, C C D C, C D C C, C D C D$.

I. Suppose $Z^{4}$ occurs in $D C C D$. Then it contains the base $C C$ of the occurrence $D * C C * D$. We have obvious inequalities $2 \partial(C)<4 \partial(Z)<2 \partial(D)+2 \partial(C)$. Therefore $\partial(Z)<\partial(C)$ and $\partial(Z)+\partial(C)<2 \partial(C)$. By Lemma 2.3 we obtain 
$C=Z^{p}, p \geq 1$. According to Lemma 2.7 we have $p \nsupseteq 2$, and using $\partial(D)<\partial(C)=$ $\partial(Z)$ we obtain $p \neq 1$.

II. Suppose $Z^{4}$ occurs in $C D C C=E D D E D E D$. Then it contains the base $D E D=D C$ of the occurrence $E D * D E D * E D$. We have $\partial(Z)<\partial(C)$. Let us consider the following cases:

(1) If $Z^{4}$ contains the suffix $E$ of the base of the occurrence $E D * D E D E * D$, then from $\partial(Z)<\partial(D)+\partial(E)=\partial(C)$ follows $(D E)^{2}=Z_{1}{ }^{t_{1}} Z_{1}{ }^{\prime}, t_{1} \geq 2$ for some cyclic shift $Z_{1}$ of $Z$. According to Lemma 2.3 we obtain $D E=$ $Z_{1}{ }^{p}, p \geq 1$. Hence $E D=C=Z_{2}{ }^{p}$. By Lemma 2.7 we have $p \nsupseteq 2$ and by $\partial(Z)<\partial(C)$ the inequality $p \neq 1$ holds.

Thus, we can assume that $Z^{4}$ is contained in the base EDDEDE of the occurrence $* E D D E D E * D$ in a word $C D C C$ and, at the same time, contains the base of the occurrence $E D * D E D * E D$.

(2) If $Z^{4}$ contains the base $D D E D$ of the occurrence $E * D D E D * E D$, then from equality $D D E D=F E D E D=F C C$, where $F$ is a suffix of $C$, and inequality $\partial(Z)<\partial(C)$ follows that $Z_{1}{ }^{\prime} Z_{1}{ }^{t_{1}}=F C^{2}, t_{1} \geq 2$ for some cyclic shift $Z_{1}$ of $Z$. Therefore $C=E D=Z_{2}{ }^{p}$, for some cyclic shift $Z_{2}$ of $Z$. Since $\partial(C D C C)<4 \partial(C)$, the case $p=1$ is impossible, and $p>1$. This contradicts the Lemma 2.7 .

(3) Thus, one can assume that $Z^{4}$ occurs in the base $D D E D E$ of occurrence $E * D D E D E * D$ and at the same time does not contain the prefix $D$ and suffix $E$ of that base. Then $Z^{4}$ occurs in $\bar{A}=\overline{E D D E D}$. This contradicts the inductive assumption.

III. Now let $Z^{4}$ occur in $C D C D=E D D E D D$. Then it contains the base $D E D=D C$ of the occurrence $E D * D E D * D$. First note that $Z^{t} \neq C D C D$ holds, since in the contrary case $\partial(Z)<\partial(C D)$, hence $C D=Z^{p}, p \geq 2$. Thus, $B=D C=Z_{1}{ }^{p}$ for some cyclic shift $Z_{1}$ of $Z$. This is a contradiction to inductive assumption. According to the case above the word $Z^{4}$ does not occur in the base $D D E D$ of the occurrence $E * D D E D * D$ since that base is equal to the base of the occurrence $E * D D E D * C$ in an already considered word $C D C C$. Let us consider the following cases:

(1) If $Z^{4}$ contains the base of the occurrence $E F * E F E E F E * F E$ in a word $C D C D$ then EFEEFE $=(C)^{2}=Z_{1}^{t_{1}} Z_{1}{ }^{\prime}$ and in view of $\partial\left(Z_{1}\right)<\partial(C)$ we have $C=Z_{1}{ }^{p}$. This contradicts the Lemma 2.7 for $p \geq 2$ and the inequality $\partial(D)<\partial(C)$ for $p=1$.

(2) Thus $Z^{4}$ occurs in the base FEEFEFE of the occurrence EFE* $F E E F E F E *$ in a word $C D C D$ and contains the base of the occurrence $E F E * F E E F E * F E$. Then $\partial(Z)<\partial(E)+\partial(F)$ holds. If $Z^{4}$ contains the base of the occurrence $E F E * F E E F E F * E$ then the word $E(E F)^{2}=G F E F E F$ is periodic with period $E F$ and $E(E F)^{2}=Z_{1}{ }^{\prime} Z_{1}{ }^{t_{1}}$. According to Lemma 2.3 we have $E F=Z_{1}{ }^{p}$ and $F E=Z_{2}{ }^{p}=D$. In view of Lemma $2.7 p \not 2$ holds and by $\partial(Z)<\partial(E)+\partial(F)$ we have $p \neq 1$. 
(3) It remains to consider the case when $Z^{4}$ strictly occurs in the base of the occurrence $E F * E F E E F E F * E$ and at the same time does not contain the prefix $E$ and suffix $F$ of that base. Then $\partial(Z)<\partial(F E)$ and $E E F E=G(F E)^{2}=Z_{1}{ }^{\prime} Z_{1}{ }^{t_{1}}$ where $t_{1} \geq 2$. According to Lemma 2.3 we have $D=F E=Z_{2}{ }^{p}$ for some cyclic shift $Z_{2}$ of $Z$, in spite of Lemma 2.7.

IV. Now suppose $Z^{t}$ occurs in a word $C C D C$. Then it contains the base $E D D=$ $C D$ of the occurrence $E D * E D D * E D$ in a word $C C D C$. Since $\partial(D)<\partial(C)$ we have $\partial(Z)<\partial(C)$. Therefore $Z_{1}{ }^{\prime} Z_{1}{ }^{t_{1}}=E D D=C D$ for some cyclic shift $Z_{1}$ of $Z$, where $t_{1} \geq 2, E$ is a suffix of $D$ and $\partial\left(Z_{1}\right)<\partial(D)+\partial(E)$. According to Lemma 2.3 we obtain that $D=Z_{1}^{p}, p \geq 1$. By Lemma 2.7 we have $p=1$ and $Z_{1}=D$. The base of the occurrence $E D * E D D * E D$ in a word $C C D C$ is non-continuable to the right relative to period $Z_{1}=D$ since the first letters of words $D$ and $E$ are different by definition of words $X_{k}$. Then $Z^{t}$ occurs in a word $\bar{A}=\overline{C C D}=\overline{E D E D D}$ that contradicts the inductive assumption.

Thus we have proved that no word of form $Z^{4}$ occurs in a subword of length four of cyclic word $\overline{D C C D C}$ over the alphabet $\{C, D\}$. Let us now prove that it does not occur in a cyclic word $\overline{D C C D C}$ either. Assuming the contrary we obtain that $Z^{t}$ contains one of the words $C C D, C D C, D C C$ and $D C D$. In view of obvious inequalities $2 \partial(C)<2 \partial(D)+\partial(C)$ and $\partial(C)+\partial(D)<2 \partial(C)+\partial(D)$ we obtain that more than half of the word $Z^{4}$ occurs in one of the following words $C C D, C D C, D C C, D C D$.

(1) Let $Z^{4}$ contain the base of the occurrence

$$
D * C C D * C=D * E H G G F E F E F E * C
$$

in a word $D C C D C$. Since $\partial(D)+\partial(E H)+\partial(C) \leq \partial(G G F E F E F E)$, we have $G G(F E)^{3}=Z_{1}{ }^{\prime} Z_{1}{ }^{t_{1}}$ for some cyclic shift $Z_{1}$ of $Z$, where $t_{1} \geq 2$. Using the Lemma 2.3 we obtain that $D=F E=Z_{2}{ }^{p}$ for some cyclic shift $Z_{1}$ of $Z$. By Lemma 2.7 we have $p=1$ and $D=Z_{2}$. Since the first letters of $C$ and $D$ are different, the word $D * E H G G F E F E F E * C$ is non-continuable to the right relative to period $D=Z_{2}$. Then $D^{4}$ is a suffix of the occurrence $* D C C D * C=* D E F G F E F E F E * C$. Therefore $F G$ is a suffix of the word $D=F E=F G F$, hence $F G=G F$. By Lemma 2.2 we get $E=T^{p}$ for some word $T$ and $p \geq 2$. This contradicts the Lemma 2.7.

(2) Suppose $Z^{4}$ contains the base of the occurrence $C * D C C * D$ in a cyclic word $C D C C D$. Since $D$ is a suffix of $C$, we have $D C C=Z_{1}{ }^{\prime} Z_{1}^{t_{1}}$ for some cyclic shift $Z_{1}$ of $Z$, and, obviously, $t_{1} \geq 2$ holds. Then, according to Lemma 2.3, the word $C=Z_{2}{ }^{p}$ is a proper power. By Lemma 2.7 we have $p=1$ and $C=Z_{2}$. But since $C$ is non-continuable to the right and to the left side one cannot count the word $C^{4}$ because of the inequality $\partial(D)<\partial(C)$, we obtain a contradiction.

(3) Let $Z^{4}$ contain the base of the occurrence

$$
C * C D C * D=E F E * G H G F(E F E)^{2} * F E
$$


or

$$
D * C D C * C=F E * G H G F(E F E)^{2} * E F E
$$

in a cyclic word $C C D C D$ or $D C D C C$ respectively. One has $E=G F=$ $G H G$. In view of the obvious inequality

$$
\partial\left(F(E F E)^{2}\right)>\partial(E F E)+\partial(F E)+\partial(E)
$$

by Lemma 2.3 and Lemma 2.7 we obtain $C=E F E=Z_{2}$. The suffix $C$ of the base of the occurrence $C * C D C * D$ is not continuable to the right relative to period $C$ because the first letters of words $C$ and $D$ are different. In view of the inequality $\partial(D)<\partial(C)$ the base of the occurrence $* C C D C * D$ does not end with word $C^{4}$. Now let $Z^{4}$ contain the base of the occurrence $D * C D C * C$. Consider the maximal power of the word $C$ that occurs in a word $D C D C C$, where one $C$ of that power coincides with the suffix $C$ of the base of the occurrence $D * C D C * C$. We can continue the occurrence $D * C D C * C$ to the right relative to period $C=Z_{2}$. Now let us count from right to left the maximal power of $C$ that occurs in a word $D C D C C$. We have the equalities

$$
D C D C C=F E E F E F E E F E E F E=F E E F C C C .
$$

It is obvious that the equality $E E F=E F E=C$ has to hold, and therefore $E F=F E$. Then $D=F E=T^{p}, p \geq 2$ that contradicts the Lemma 2.7.

(4) Finally suppose $Z^{4}$ contains the base of the occurrence

$$
C * D C D * C=E F * E D C D * C
$$

in a cyclic word $C D C D C$. Let us repeat the reasoning of case one. Having changed only the occurrence $D * C C D * C$ by the occurrence $E F * E D C D *$ $C=E F * C C D * C$ in a word $C D C D C$, we can assume, that $Z^{4}$ does not contain the base of the occurrence $E F * C C D * C$. Therefore $Z^{4}$ occurs in a base of the occurrence $E F * E D C D C *$ and at the same time contains the base of the occurrence

$$
E F E * D C D * C=E F E * G F E D D * C=E F E * G D D D * E D .
$$

We have the inequality $2 \partial(D)+\partial(E) \leq \partial\left(G D^{3}\right)$. Then $G D^{3}=Z_{1}{ }^{\prime} Z_{1}{ }^{t_{1}}$ for some cyclic shift $Z_{1}$ of $Z$, and $t_{1} \geq 2$. Hence $D=Z_{2}{ }^{p}$. By Lemma 2.7 we have $p=1$ and $D=Z_{2}$. Since the first letters of words $C$ and $D$ are different, the occurrence $E F E * G F E D D * C$ is not continuable to the right relative to period $D$. If $D^{4}$ is a suffix of the base of occurrence $* C D C D * C=* C F G F E D D * C$ then from right to left we read $D^{2}, D=F E$, and the equality $F G=G F=E$ must hold. Therefore $E=T^{p}, p \geq 2$ that contradicts the Lemma 2.7. Thus we proved that 
no word of form $Z^{t}, t \geq 4$ can occur in a word $\overline{X_{k+1}}=\overline{D C C D C}$. The Lemma is proved completely.

It remains to note that the Lemma 2.8 is a reformulation of Proposition 1.2.

\section{Proof of the MAIN Result}

Now turn to the proof of Theorem 1.1.

Proof. Suppose that $\varphi$ has a finite order in $\operatorname{Aut}(B(2, n))$, that is $\varphi^{k}=i d$. Then, particularly $\varphi^{k}(a)=a$, that is the word $X_{k}=a$. Therefore $a^{-1} X_{k}$ is equal to the empty word in $B(2, n)$. Consider two possible cases:

(1) If $X_{k}$ starts with the letter $b$, then $a^{-1} X_{k}$ is not reducible and obviously contains no forth power of a non-empty word by Proposition 1.2 since all letters in $X_{k}$ are positive.

(2) If $X_{k}$ starts with the letter $a$, then we reduce $a^{-1} a$ and the result $X_{k}{ }^{\prime}$ contains no fourth power by Lemma 1.2. Hence, by definition 2.5 the irreducible word $a^{-1} X_{k}$ is absolutely reduced for odd $n \geq 665$ and according to Lemma 2.4 it cannot be equal to the empty word in $B(2, n)$. This proves the Theorem 1.1 for odd $n$.

To prove the Theorem 1.1 for even $n=16 k \geq 8000$ (as in [4]) we use Theorem 2(i) of [8] according to which if a non-empty freely non-reducible word $X$ is equal to one in $B(m, n)$, then $X$ contains a non-empty subword of the form $A^{(n / 2)-1240}$. Again by Proposition 1.2 the word $a^{-1} X_{k}$ contains no subword of the form $A^{n / 2-1240}$, hence it cannot be equal to the empty word in $B(2, n)$.

\section{Appendix}

The author thanks the Referee for suggesting the following much shorter proof of Proposition 1.2 using some well-known properties of Fibonacci words.

Proof. Let $h$ denote the Fibonacci morphism given by

$$
h: a \mapsto b, \quad h: b \mapsto b a .
$$

It is well-known that the reversal of $h(b)$ is a conjugate of $h(b)$ for all $k \geq 0$. Thus the square of the reversal of $h^{n-1}(b)$ is a factor of the cube $h^{n-1}(b)^{3}$, which is a factor of the Fibonacci infinite word $\lim _{k \rightarrow \infty} h^{k}(b)$ for all $n \geq 3$. Therefore the square of $h^{n-1}(b)$ does not contain 4th powers (see [6], Thm. 2). The claim now follows from the fact that the word $\varphi^{n}(a)$ equals the reversal of $h^{n-1}(b)$, which can be proved by induction.

Acknowledgements. The author is grateful to the anonymous referees for detailed review of the paper and helpful suggestions. 


\section{REFERENCES}

[1] S.I. Adian, The Burnside Problem and Identities in Groups. Nauka (1975); English translation, Springer-Verlag (1979) 1-336.

[2] V.S. Atabekyan, Non- $\varphi$-admissible normal subgroups of free Burnside groups. J. Contemp. Math. Anal. 45 (2010) 112-122.

[3] V.S. Atabekyan, Normal automorphisms of free Burnside groups. Izv. RAN. Ser. Math. 75 (2011) $3-18$

[4] E.A. Cherepanov, Free semigroup in the group of automorphisms of the free Burnside group. Comm. Algebra 33 (2005) 539-547.

[5] E.A. Cherepanov, Normal automorphisms of free Burnside groups of large odd exponents. Int. J. Algebra Comput. 16 (2006) 839-847.

[6] J. Karhumäki, On cube-free $\omega$-words generated by binary morphisms. Disc. Appl. Math. 5 (1983) 279-297.

[7] Kourovka Notebook, Unsolved Problems in Group Theory. Novosibirsk (2006).

[8] I.G. Lysënok, Infinite Burnside groups of even exponent. Izv. Math. 60 (1996) 453-654.

[9] F. Mignosi and G. Pirillo, Repetitions in the Fibonacci infinite word. Informatique Théorique et Applications 26 (1992) 199-204.

Communicated by J. Karhumäki.

Received April 12, 2010. Accepted March 1st, 2011. 